\title{
Un análisis tipológico del estrés parental en familias en riesgo psicosocial
}

\author{
Javier Pérez Padilla, ${ }^{1}$ Susana Menéndez Álvarez-Dardet ${ }^{1}$
}

Artículo original

\section{SUMMARY}

The aim of this study was to explore the level and type of both parental stress and coping strategies in a sample of 109 mothers from at-risk families attended by Social Services. A cluster analysis revealed three groups: Adapted-Strategic, Clinical-Avoidant, and Extreme-Passive. A multinomial logistic regression analysis showed that parental sense of competence, parental locus of control and family cohesion influenced the probability of inclusion of the mothers in each group. Implications for intervention strategies are discussed.

Key words: Parenting stress, coping, sense of competence, parental locus of control, family cohesion.

El estrés parental constituye un complejo proceso en el que los progenitores se sienten desbordados ante las demandas que deben afrontar en su papel como padres o madres. ${ }^{1}$ De acuerdo con Abidin, ${ }^{2}$ esta dimensión psicológica se caracteriza por ser un elemento activador que fomenta la utilización de los recursos disponibles para afrontar de una manera satisfactoria el ejercicio del papel parental, siendo tan perjudicial la falta absoluta de activación como unos niveles extremos de estrés. Enmarcado dentro del modelo general de estrés de Lazarus y Folkman, ${ }^{3}$ el modelo de estrés parental de Abidin ${ }^{2}$ plantea que la sensación de desbordamiento se basa tanto en una autoevaluación de los recursos propios frente a las demandas parentales, como en la puesta en marcha de estrategias de afrontamiento para solventar las dificultades asociadas a la tarea de educar a los hijos. Abidin distingue entre dos componentes específicos: el estrés asociado en general a las exigencias parentales y el que se deriva específicamente del propio hijo o hija. ${ }^{4}$

Una dimensión fundamental a la hora de analizar el estrés parental está constituida por las estrategias de afrontamiento que los progenitores utilizan para hacerle frente,

\section{RESUMEN}

Este estudio examina el grado y tipo de estrés parental y de estrategias de afrontamiento en una muestra de 109 madres en situación de riesgo con un expediente activo en los Servicios Sociales Comunitarios. Los análisis de conglomerados realizados mostraron tres perfiles diferenciados: Ajustado-Estratégico, Clínico-Evitativo y Extremo-Pasivo. Un análisis de regresión logística multinomial indicó que el sentimiento de competencia parental, el locus de control como progenitor y la cohesión familiar influían en la pertenencia de las madres a cada uno de los grupos. Se discuten las implicaciones prácticas de estos resultados de cara a la intervención con estas familias.

Palabras clave: Estrés parental, afrontamiento, sentido de competencia, locus de control parental, cohesión familiar. y que suponen un conjunto de procesos cognitivos o conductuales desplegados con objeto de tratar las demandas concretas de una situación percibida como desbordante o que sobrepasa a los recursos personales. ${ }^{3}$ Existen numerosas propuestas de clasificación de estos procesos, desde la agrupación en comportamientos activos vs. evitativos hasta la conceptualización de más de 100 estrategias. ${ }^{5}$ Cabe apuntar que el hecho de utilizar uno o varios tipos de afrontamiento por sí solos no reduce el estrés, por lo que las diferentes estrategias no pueden calificarse a priori como adaptativas o desadaptivas. ${ }^{6}$ Sin embargo, el uso de estrategias activas puede fortalecer a los progenitores y a la familia ante situaciones estresantes, siendo la evitación un indicador de alteraciones psicológicas. ${ }^{78}$

Existe mucha bibliografía internacional sobre el estrés parental y las evidencias acumuladas señalan que una elevada sensación de desbordamiento se relaciona con indicadores negativos tanto en los progenitores como en sus hijos. Así, diversos estudios han documentado que los padres y las madres con elevados niveles de estrés parental tienden a presentar síntomas depresivos, ansiedad, un locus de control ex-

Departamento de Psicología Evolutiva y de la Educación. Universidad de Huelva, España.

Correspondencia: Javier Pérez Padilla. Departamento de Psicología Evolutiva y de la Educación. Universidad de Huelva. Campus del Carmen. Av. Tres de Marzo s/n. 21071 Huelva, España. Tel. (+34) 959 21-9208 Fax. (+34) 959 21-9201. E-mail: javier.perez@dpee.uhu.es

Recibido: 19 de junio de 2013. Aceptado: 29 de octubre de 2013. 
terno y suelen desplegar prácticas educativas disfuncionales e incluso maltratantes., ${ }^{9,10}$ En la misma línea, aquellos menores que conviven con padres muy estresados tienden a tener más problemas de internalización y externalización y dificultades en la regulación emocional. ${ }^{11,12}$ Por tanto, el estrés parental se ha erigido como una dimensión relevante para el estudio de la familia y de las interacciones entre sus componentes; no obstante, los estudios sobre el estrés parental tienden a basarse en el grado del mismo, realizándose de forma habitual acercamientos de tipo cuantitativo, en detrimento de análisis centrados no tanto en el grado como en las tipologías de los progenitores y cómo éstos lo experimentan.

De acuerdo con diferentes estudios, la vivencia del estrés se asocia tanto a dimensiones individuales de los padres como a la dinámica familiar. Respecto a las primeras, el análisis de la percepción del papel (rol) como progenitor ha resultado ser especialmente relevante. Así, el sentimiento de competencia parental se relaciona con la vivencia del estrés de tal forma que la eficacia percibida como padre o madre puede funcionar como un elemento protector frente al estrés. ${ }^{13-15}$ La satisfacción parental es una dimensión altamente relacionada con la percepción de eficacia y puede propiciar una respuesta adaptativa a la tensión percibida por los padres. ${ }^{16}$ Por otro lado, el afrontamiento del estrés también está influido por el sentimiento de competencia parental. Tal como apuntan Jones y Prinz, ${ }^{13}$ existen distintas investigaciones que asocian el afrontamiento pasivo al efecto conjunto de la baja percepción de eficacia como progenitor y a un estilo de atribución causal de tipo externo. Por su parte el locus de control parental, entendido como las expectativas del padre o la madre en cuanto a las contingencias de su comportamiento y las consecuencias observadas en relación a las tareas propias de su rol, ${ }^{17}$ se ha relacionado tanto con el estrés como con las estrategias utilizadas para afrontarlo. Así, ante situaciones percibidas como controlables el uso de estrategias de afrontamiento activas tiende a reducir el estrés., ${ }^{5,17}$ Además, los progenitores con un locus de control interno suelen presentar menores niveles de estrés y mejores indicadores de bienestar psicológico en situaciones de dificultad. ${ }^{19,20}$

Junto a las dimensiones individuales de la maternidad y la paternidad, algunas facetas de la dinámica familiar también influyen en la percepción de desbordamiento de los progenitores. Así, la cohesión familiar (es decir, unos lazos afectivos moderadamente fuertes entre los componentes de la familia), tiende a amortiguar los efectos negativos derivados de un elevado estrés en los padres. Este vínculo emocional fue estudiado por Amerikaner, Monks, Wolfe y Thomas ${ }^{21}$ para explorar la salud psicológica de los miembros de la familia, erigiéndose como un factor clave y determinante. En la misma línea, y de acuerdo con Ramírez, Manongdo y Cruz-Santiago, ${ }^{22}$ una cohesión familiar satisfactoria tiene un efecto amortiguador en ambientes estresantes y violentos. Con respecto al afrontamiento, según Hardy, Power y Jaedicke ${ }^{23}$ el apoyo afectivo entre los miembros de la familia se relaciona con el tipo y número de estrategias utilizadas por los hijos, especialmente con menos evitación ante situaciones incontrolables.

Por tanto, el estrés parental influye tanto en el ajuste de los padres como en el de los hijos, encontrándose relacionado con las percepciones que tienen de sí mismos los progenitores y con las dinámicas que se desarrollan en la familia. No obstante, aunque el análisis del estrés parental puede aportar información relevante, los estudios centrados en el mismo suelen tener un carácter descriptivo, relacional y, en menor medida, explicativo. Además, tal como se ha comentado anteriormente, existen escasos acercamientos tipológicos que, más allá de analizar el grado de estrés, ofrezcan claves acerca de las diferentes formas de experimentarlo y afrontarlo, y a su vez sobre cómo intervenir y trabajar con estos padres de una manera adaptada a sus necesidades. Hay algunas evidencias sobre la pertinencia y el interés de un análisis tipológico del estrés, como por ejemplo los trabajos de Krohne, ${ }^{24}$ en los que distinguen cuatro tipologías de personas con respecto al estilo de afrontamiento que despliegan.

Las familias en riesgo son un colectivo específico caracterizado por niveles importantes de estrés como consecuencia de las circunstancias adversas que experimentan, tanto personales como del entorno en el que se desenvuelven. ${ }^{25}$ Estas familias tienen dificultades importantes para atender de manera adecuada las necesidades de los menores, pero sin alcanzar una gravedad que justifique la separación de éstos de sus padres. ${ }^{26}$ La bibliografía internacional sobre estas familias señala que los progenitores que las encabezan se encuentran altamente estresados y desbordados, llegando a extremos clínicos. ${ }^{27} \mathrm{Al}$ igual que sucede en los estudios sobre familias no declaradas en riesgo resumidos más arriba, existen diferentes dimensiones tanto individuales como familiares asociadas al estrés parental en las familias en situación de adversidad. Con respecto a las dimensiones de índole cognitiva relacionadas con el rol como progenitor, diversos estudios señalan que muchos de estos padres y madres tienden a percibirse como muy eficaces a la hora de autoevaluarse como tales, debido en gran medida a las atribuciones de tipo externo que tienden a realizar al explicar las causas de los problemas de sus hijos. ${ }^{13,26}$ En cuanto a la dinámica familiar, en una muestra de mujeres que vivían en condiciones de pobreza y que acudían a programas de apoyo familiar, Vandsburger, Harrigan y Biggerstaff ${ }^{28}$ hallaron que la cohesión promovía la resiliencia del sistema ante la acumulación de factores estresantes. En la misma línea, en la revisión de Zolkoski y Bullock ${ }^{29}$ se señala que la cohesión familiar promueve la resiliencia de los menores en entornos de pobreza, violencia y abuso de sustancias. De nuevo, al igual que en la bibliografía más general sobre el estrés parental, en las investigaciones sobre familias en riesgo ha primado un abordaje cuantitativo, centrado en examinar el grado de estrés relacionado con el rol de progenitor. No obstante, existen algunas evidencias que apoyan la conveniencia de complementar este enfoque con análisis tipológicos en el 
estudio del estrés en familias en riesgo. Por ejemplo, Ayoub, Willet y Robinson ${ }^{30}$ identificaron cinco grupos en función de la vivencia y los tipos de estresores que experimentaban: familias estresadas situacionalmente, crónicamente, emocionalmente, multiestresadas y multiestresadas con violencia. Este tipo de agrupaciones es útil para que distintos tipos de familias puedan beneficiarse de intervenciones adaptadas a sus necesidades y fortalezas. ${ }^{31}$

Por tanto, las dimensiones descritas en este trabajo tienen interés por sus influencias en el complejo proceso de estrés que experimentan padres y madres tanto en general como específicamente en aquellas familias que se encuentran en situación de adversidad y que, en casos extremos, pueden ser un contexto de maltrato infantil. Siguiendo las conclusiones del meta-análisis de Stith et al., ${ }^{32}$ la cohesión familiar disfuncional y altos niveles de estrés parental son relevantes factores de riesgo para el maltrato físico y la negligencia. En la misma línea, el modelo de afrontamiento al estrés del maltrato infantil de Hillson y Koupier ${ }^{33}$ plantea que el uso de estrategias de afrontamiento desadaptativas (retirada conductual/cognitiva, focalización en el estado de irritabilidad...), puede desembocar en negligencia o maltrato físico. No obstante, la investigación sobre las intervenciones con familias en riesgo o con colectivos deprimidos señala que la percepción de estrés parental es una dimensión con la que se puede trabajar y que, además, puede reducirse a niveles óptimos. ${ }^{34-36}$ En España existen pocas investigaciones sobre estrés parental en familias en riesgo, sin embargo los resultados preliminares de Padilla, Máiquez y Rodrigo ${ }^{37,38}$ señalan que esta dimensión es sensible a la intervención familiar así como un indicador fiable de su eficacia. Asimismo, las dimensiones consideradas en este estudio son susceptibles de ser modificadas por medio del trabajo con las familias, reduciendo así la percepción de desbordamiento y malestar en los progenitores. ${ }^{15,28,35}$ Sin embargo, aunque existe un creciente interés por caracterizar y analizar diferentes dimensiones psicosociales presentes en las familias en riesgo en España, ${ }^{25,26,31,39}$ hay pocos estudios en los que se hayan examinado los constructos expuestos, a pesar de que han mostrado su relevancia en la bibliografía internacional.

Este trabajo se centra en el análisis del grado y tipo de estrés parental experimentado por los progenitores de familias en situación de riesgo usuarias de los Servicios Sociales Comunitarios. En concreto, este estudio pretende cubrir los siguientes objetivos:

1. Realizar un examen descriptivo y tipológico del estrés parental experimentado por estos progenitores y de las estrategias de afrontamiento que despliegan para hacerle frente.

2. Analizar las relaciones entre el grado y el tipo de estrés $\mathrm{y}$ algunas dimensiones individuales y grupales relativas a la maternidad y la paternidad: el sentimiento de competencia parental, el locus de control parental y la cohesión familiar.

\section{MÉTODO \\ Participantes}

La muestra estaba formada por 109 madres de familias en situación de riesgo con un expediente activo en los Servicios Sociales Comunitarios de la diputación y la ciudad de Huelva, España. El criterio de inclusión fue que la familia se ajustara a un perfil de riesgo medio que es el característico de aquellas con las que se desarrollan intervenciones de preservación y fortalecimiento familiar. La edad de estas mujeres se situó en torno a los 35 años $(M=35.35, D T=7.25)$, y su nivel educativo era principalmente bajo: la mayoría o bien no había completado la enseñanza básica (42.6\%) o bien tenía estudios primarios (21.8\%). Un 36.1\% de las madres trabajaba de manera remunerada, pero los importantes niveles de inestabilidad (50\%) y de falta de contrato $(45.2 \%)$ indican una notable precariedad laboral. Las familias estaban compuestas por aproximadamente cuatro personas ( $D T=1.26)$ y dos-tres hijos o hijas $(M=2.41, D T=1.20)$, y eran monoparentales en un $40.2 \%$ de los casos. La comparación de sus ingresos anuales (corregidos por unidad de consumo) con los datos oficiales poblacionales de cada año revela que el $56 \%$ de estas familias vivían por debajo del umbral de la pobreza. ${ }^{40}$

\section{Instrumentos}

Los datos que se presentan en este trabajo se han obtenido utilizando las escalas que se describen a continuación. En todos los casos se trata de instrumentos autoadministrados que aportan resultados que indican un mayor nivel de la dimensión mientras más elevado sea el valor obtenido.

- Estrés parental (PSI-SF). Esta prueba está compuesta por 36 reactivos con una escala Likert de cinco opciones de respuesta, y evalúa el nivel de estrés experimentado por la persona en su rol como progenitor. La adaptación española ${ }^{41}$ revela una estructura bifactorial y recomienda criterios de corrección que permiten obtener, junto a una puntuación final de estrés parental, resultados sobre dos subescalas: estrés asociado en general a la paternidad o la maternidad (malestar personal, 12 reactivos) y específicamente a la crianza del propio hijo o la hija (estrés derivado del cuidado del hijo, 24 reactivos). Evaluada mediante el índice alpha de Cronbach, la fiabilidad de esta prueba en esta muestra fue $\alpha=.89$ para la puntuación total y $\alpha=.79$ y $\alpha=.85$ para las subescalas de malestar parental y estrés derivado del cuidado del hijo, respectivamente.

- Estrategias de afrontamiento del estrés (COPE-AS). ${ }^{42}$ Esta escala evalúa las estrategias que se utilizan para hacer frente a diferentes situaciones problemáticas relacionadas con el cuidado de los hijos, y está compuesta por 28 reactivos con cuatro opciones Likert de respuesta. La adaptación a nuestro país por Crespo y Cruzado ${ }^{43}$ 
ofrece resultados de tres subescalas que aportan información sobre las estrategias de afrontamiento centradas en el problema (seis reactivos, $\alpha=.73$ ), en la emoción (10 reactivos, $\alpha=.50$ ) y en la evitación (seis reactivos, $\alpha=.67$ ).

- $\quad$ Sentimiento de competencia parental (PSOC). ${ }^{44}$ La prueba explora la percepción que los progenitores tienen acerca de su capacidad como padres y la satisfacción experimentada en el desempeño de su rol. Está formada por 16 reactivos con una escala Likert de seis opciones, y aporta información sobre la eficacia percibida como progenitor (siete reactivos, $\alpha=.73$ ) y la satisfacción con el rol parental (nueve reactivos, $\alpha=.50$ ).

- Locus de control parental (PLOC). ${ }^{17}$ Esta escala evalúa, mediante 47 reactivos $(\alpha=.71)$, el tipo de atribución que las madres y los padres hacen acerca de determinadas conductas infantiles. Cada frase se responde mediante una escala Likert de cinco opciones y aporta una puntuación global que refleja un locus de control externo o interno en sus valores más altos o bajos respectivamente.

- Cohesión familiar. Se utilizó la escala correspondiente de FACES-III ${ }^{45}$ formada por 10 reactivos con cinco opciones Likert de respuesta, sobre la unión emocional y los lazos afectivos que existen entre los miembros de la familia. La fiabilidad en este estudio ha sido de $\alpha=.79$.

\section{Procedimiento}

El equipo de investigación mantuvo una reunión con las psicólogas y los psicólogos de los Servicios Sociales Comunitarios para exponerles los objetivos del estudio, las características de la población blanco, y su participación en el trabajo de campo. Tras esta reunión, el 62.5\% de los profesionales de estos equipos de intervención psicosocial mostraron su disposición a colaborar. Cada técnico seleccionó un grupo de usuarias de su centro y, después de recabar su colaboración voluntaria en el estudio, concertó una cita en las dependencias del centro social a la que acudió un miembro del equipo de investigación, para administrar una batería de instrumentos de evaluación del contexto familiar que incluía las escalas que se acaban de describir.

\section{RESULTADOS}

\section{Examen descriptivo y bivariante de las dimensiones}

La puntuación del estrés parental alcanzó una media (cuadro 1) que se situó por encima del valor crítico a partir del cual los autores de la escala establecen que puede hablarse de puntuaciones extremas de estrés (86.4), ${ }^{4}$ de manera que el $45.95 \%$ de la muestra se caracterizó por niveles extremos y el $15.6 \%$ por niveles clínicos. Tanto el malestar parental como el estrés derivado del cuidado del niño guardaron relación con la mayoría de las dimensiones estudiadas. Así, una mayor frecuencia de conductas evitativas, un locus de control externo, un menor sentido de eficacia y menores niveles de satisfacción, así como una cohesión familiar más baja se relacionaron con mayores sentimientos de malestar asociado al papel de progenitor. La estrategia de afrontamiento centrada en el problema se relacionó positivamente con la cohesión familiar mientras que la evitación lo hizo con el locus de control externo y la insatisfacción. Con respecto a las dimensiones personales y familiares, el locus de control parental se correlacionó de manera negativa con la eficacia y la satisfacción como progenitor, mientras que las variables familiares no se asociaron con las individuales de manera significativa.

\section{Análisis tipológico de los indicadores de estrés}

Con objeto de realizar un examen tipológico de los diversos indicadores de estrés se llevó a cabo un análisis de conglomerados. Este procedimiento estadístico multivariante permite clasificar un conjunto de casos en grupos (conglomerados) heterogéneos entre sí pero homogéneos a nivel interno, ${ }^{46,47}$ de manera que la similitud en función de las dimensiones consideradas se maximiza dentro de cada grupo y se minimiza entre los grupos. ${ }^{48}$ Aunque esta técnica estadística es muy robusta ante el incumplimiento de los supuestos previos de la estadística paramétrica, ${ }^{46}$ según Pérez ${ }^{47}$ antes de

Cuadro 1. Correlaciones entre los indicadores de estrés y dimensiones personales y familiares

\begin{tabular}{|c|c|c|c|c|c|c|c|c|c|}
\hline & $M(D T)$ & 2 & 3 & 4 & 5 & 6 & 7 & 8 & 9 \\
\hline 1. Estrés parental - malestar & $31.94(10.32)$ & $.555^{* * *}$ & .048 & .115 & $.266^{* *}$ & $.421^{* * *}$ & $-.225^{*}$ & $-.354^{* * *}$ & $-.265^{* *}$ \\
\hline 2. Estrés parental - hijos & $62.14(16.76)$ & - & .112 & .137 & .154 & $.545^{\star * *}$ & $-.209 *$ & $-.426 * * *$ & $-.245^{\star}$ \\
\hline 3. Afrontamiento - problema & $17.04(4.26)$ & & - & $.502 * * *$ & .114 & -.067 & -.110 & -.035 & $.269 * *$ \\
\hline 4. Afrontamiento - emoción & $21.21 \quad(5.24)$ & & & - & $.331 * *$ & .013 & .188 & -.090 & .192 \\
\hline 5- Afrontamiento - evitación & $11.48 \quad(3.97)$ & & & & - & $.314^{* *}$ & .046 & $-.217^{\star}$ & -.101 \\
\hline 6. Locus de control parental & $115.62(18.61)$ & & & & & - & $-.259 * *$ & $-.303 * * *$ & -.178 \\
\hline $\begin{array}{l}\text { 7. Sentido de eficacia } \\
\text { parental }\end{array}$ & $29.24(6.91)$ & & & & & & - & .153 & .087 \\
\hline 8. Satisfacción parental & $30.00 \quad(6.90)$ & & & & & & & - & .000 \\
\hline 9. Cohesión familiar & $37.51 \quad(7.94)$ & & & & & & & & - \\
\hline
\end{tabular}

${ }^{*} p<.05 ;{ }^{* *} p<.01 ;{ }^{* *} p<.001$. 
Tipología del estrés parental

Cuadro 2. Descriptivos y contrastes de medias entre los conglomerados en función de los indicadores de estrés

\begin{tabular}{|c|c|c|c|c|c|c|c|c|}
\hline \multirow[b]{2}{*}{ Dimensiones } & \multicolumn{2}{|c|}{$\begin{array}{c}\text { Ajustado-Estratégico } \\
(35.1 \%)\end{array}$} & \multicolumn{2}{|c|}{$\begin{array}{c}\text { Clínico-Evitativo } \\
(22.3 \%)\end{array}$} & \multicolumn{2}{|c|}{$\begin{array}{c}\text { Extremo-Pasivo } \\
(42.6 \%)\end{array}$} & \multicolumn{2}{|c|}{ ANOVA } \\
\hline & $M$ & (DT) & $M$ & $(\mathrm{DT})$ & $M$ & $(\mathrm{DT})$ & $\mathrm{F}$ & DMS \\
\hline Estrés parental - Malestar & 24.55 & (7.68) & 44.76 & (8.79) & 31.32 & (6.88) & $41.40 * * *$ & $\begin{array}{l}1-2^{* * *} \\
1-3^{* *} \\
2-3^{* * *}\end{array}$ \\
\hline Estrés parental - Hijos & 50.611 & $(11.26)$ & 82.901 & $11.68)$ & 62.301 & $(13.40)$ & $44.23^{* * *}$ & $\begin{array}{l}1-2^{* * *} \\
1-3^{* * *} \\
2-3^{* * *}\end{array}$ \\
\hline Afrontamiento - Problema & 20.09 & $(3.40)$ & 18.00 & $(3.26)$ & 13.90 & $(3.11)$ & $34.14^{* * *}$ & $\begin{array}{l}1-2^{*} \\
1-3^{* * *} \\
2-3^{* * *}\end{array}$ \\
\hline Afrontamiento - Emoción & 24.21 & $(5.04)$ & 22.67 & $(5.03)$ & 17.97 & (3.49) & $19.30 * * *$ & $\begin{array}{l}1-2 \\
1-3^{* * *} \\
2-3^{* * *}\end{array}$ \\
\hline Afrontamiento - Evitación & 12.21 & $(4.37)$ & 14.28 & $(3.62)$ & 9.40 & $(2.56)$ & $14.34^{* * *}$ & $\begin{array}{l}1-2^{*} \\
1-3^{* * *} \\
2-3^{* * *}\end{array}$ \\
\hline
\end{tabular}

${ }^{*} p<.05 ;{ }^{* *} p<.01 ;{ }^{* *} p<.001$

realizar este tipo de análisis es importante examinar tanto la presencia de casos extremos como la existencia de problemas de linealidad. El examen de los diagramas de caja y bigotes de cada variable y el cálculo de la distancia de Mahalanobis no revelaron la existencia de casos extremos univariantes y multivariantes respectivamente, y los índices de correlación bivariada entre las diversas dimensiones (cuadro 1) no superan en ningún caso el valor $r=.80$, indicando así que no existen problemas de colinealidad. ${ }^{49}$

De acuerdo con las recomendaciones de diversos autores $^{46-48}$ se efectuaron dos tipos de análisis de conglomerados, el primero con carácter exploratorio y el segundo con fines confirmatorios; para evitar problemas derivados del diferente escalonamiento de las variables se trabajó con las puntuaciones una vez estandarizadas. En primer lugar, se computó un análisis de conglomerados jerárquico aglomerativo repitiendo el procedimiento con distintos métodos de conglomeración (en concreto vinculación intergrupos, vecino más lejano, y método de Ward); en todos los casos, el examen visual de cada dendograma reveló la existencia de tres agrupaciones diferenciadas. Para confirmar esta solución se llevó a cabo un análisis de conglomerados de k-medias solicitando la definición de tres grupos. El historial de iteraciones mostró que la convergencia (y, por tanto, la ausencia de cambios en los centros de cada conglomerado) se alcanzó en la sexta iteración.

Los datos que permiten definir de manera detallada la solución final de tres grupos se ofrecen en el cuadro 2 que recoge los resultados del análisis de varianza de esta tipología y las medias en cada conglomerado de las puntuaciones directas de las dimensiones introducidas en el análisis de conglomerados.

Tal como se puede observar en el cuadro 2, los tres grupos se formaron de manera diferencial en función de todas las dimensiones de estrés incluidas en el estudio. Así, el Grupo 1(madres Ajustadas-estratégicas) estuvo definido por niveles moderados de malestar tanto parental como derivado del cuidado del niño, por muchas estrategias de afrontamiento centradas en el problema y en la emoción y por un nivel medio de evitación. El Grupo 2 (Clínicas-evitativas), se caracterizó por niveles clínicos de estrés parental, por un afrontamiento centrado en el problema y en la emoción moderados y una alta frecuencia de la evitación. En último lugar, el Grupo 3 (Extremas-pasivas) se distinguió por niveles extremos de malestar personal y de estrés derivado del cuidado del niño, y por pocas estrategias de afrontamiento y evitativas.

\section{Determinantes personales y familiares del grado y tipo de estrés parental}

Con el objetivo de estimar la influencia de las dimensiones individuales y familiares consideradas en las tres tipologías, se realizó un análisis de regresión logística multinomial. Esta prueba es más robusta frente al incumplimiento de los supuestos habituales y ofrece más información de las variables intervinientes que el análisis discriminante gracias a los valores ORs (Odds Ratio), que indican el incremento de la probabilidad de pertenencia de un grupo por cada incremento en la unidad de la variable independiente. ${ }^{49,50}$ En el caso de este estudio, el modelo resultante de este análisis estima los factores asociados a la probabilidad de que una madre del grupo Clínico-evitativo o del Extremo-pasivo pase a pertenecer al Ajustado-estratégico, es decir, el caracterizado por mejores indicadores en cuanto al estrés parental y a las estrategias de afrontamiento utilizadas. Así, este análisis permite identificar el peso específico que cada covariable (en este estudio, 
Pérez Padilla y Menéndez Álvarez-Dardet

Cuadro 3. ANOVA entre las tres tipologías de estrés y dimensiones personales y familiares

\begin{tabular}{|c|c|c|c|c|c|c|c|c|}
\hline \multirow[b]{2}{*}{ Dimensiones } & \multicolumn{2}{|c|}{$\begin{array}{c}\text { Ajustado-Estratégico } \\
(35.1 \%)\end{array}$} & \multicolumn{2}{|c|}{$\begin{array}{c}\text { Clínico-Evitativo } \\
(22.3 \%)\end{array}$} & \multicolumn{2}{|c|}{$\begin{array}{c}\text { Extremo-Pasivo } \\
(42.6 \%)\end{array}$} & \multicolumn{2}{|c|}{ ANOVA } \\
\hline & M & (DT) & M & (DT) & M & (DT) & $\mathrm{F}$ & DMS \\
\hline Locus de control parental & 107.94 & 15.37) & 130.141 & (14.91) & 115.071 & (18.63) & $11.364^{* * *}$ & $\begin{array}{l}1-2^{* * *} \\
1-3 \\
2-3^{* * *}\end{array}$ \\
\hline Eficacia & 30.33 & (6.77) & 25.81 & $(6.85)$ & 29.50 & (6.99) & 2.982 & $\begin{array}{l}1-2^{*} \\
1-3 \\
2-3^{*}\end{array}$ \\
\hline Satisfacción & 31.61 & (6.71) & 24.86 & $(6.78)$ & 30.18 & $(6.66)$ & $6.863 * *$ & $\begin{array}{l}1-2^{* * *} \\
1-3 \\
2-3^{* *}\end{array}$ \\
\hline Cohesión & 41.91 & (4.89) & 33.85 & (7.29) & 35.53 & (8.97) & $9.767^{* * *}$ & $\begin{array}{l}1-2^{* * *} \\
1-3^{* * *} \\
2-3\end{array}$ \\
\hline
\end{tabular}

${ }^{*} p<.05 ;{ }^{* *} p<.01 ;{ }^{* * *} p<.001$

el locus de control parental, la satisfacción como progenitor, la eficacia percibida como padre o madre y la cohesión familiar), tiene a la hora de predecir la pertenencia a un grupo.

En primer lugar se realizaron análisis de varianza entre los tres grupos y las dimensiones señaladas para seleccionar qué variables se tomarían en cuenta para formar el modelo. Estos análisis se presentan en el cuadro 3, y tal como se puede observar todas resultaron ser significativas exceptuando la percepción de eficacia como madre, por lo que no se incluyó al no ser un indicador sensible a la pertenencia a los grupos. Por tanto, se utilizaron tres covariables en una muestra de 109 sujetos de manera que se cumple ampliamente el requisito de tamaño muestral de este tipo de análisis. ${ }^{51}$

Una vez seleccionadas las covariables, se introdujeron en un solo paso para establecer el modelo de regresión logística. En el cuadro 4 se presentan los factores asociados a las tipologías de estrés, tomando como referencia el grupo Ajustado-estratégico. El examen de las puntuaciones B de cada covariable, así como de las ORs, permite establecer si cada una constituye, por sí sola, un elemento que aumente la probabilidad de pertenencia a dicho grupo.

El grupo Clínico-evitativo estuvo influido por las tres variables escogidas para el modelo de regresión logística. La satisfacción parental y la cohesión familiar puntuaron de manera negativa con respecto al primer grupo, es decir, el aumento de un punto en estas covariables incrementaba respectivamente un $64 \%$ y un $77 \%$ las probabilidades de pertenencia al conglomerado Ajustado-estratégico. El locus de control parental fue la dimensión que más alto puntuó (251\% de probabilidad por cada punto) con una B negativa, en comparación con el grupo de referencia. Es decir, el aumento de la cohesión familiar y de la satisfacción parental, así como un locus de control más interno incrementaba la probabilidad de que las madres del grupo Clínico-evitativo pasaran a pertenecer al Ajustado-estratégico. Por su parte el grupo Extremo-pasivo se vio influido, en comparación con el primero, únicamente por la cohesión familiar. Esta dimensión fue la que más información aportaba al modelo con una probabilidad de pertenencia al grupo de referencia del $70 \%$ por cada punto aumentado si esta covariable fuera modificada.

La decisión de considerar el modelo final se tomó en función del principio de parsimonia que debe regir este tipo de análisis, así como otros indicadores que ofrecen información sobre la validez y el ajuste del mismo: la bondad de ajuste mediante la prueba de Chi-cuadrada, el contraste de razón de verosimilitud entre modelos y la calidad del modelo final mediante el estadístico de Nagelkerke. ${ }^{49,50} \mathrm{El}$ primer indicador de importancia que permite conocer la correspondencia del modelo con los datos es la bondad de ajuste, medido por medio de la devianza $\left(\chi^{2}=149.30, p=.913\right)$, concluyendo que el modelo es adecuado para el ajuste de los datos. La prueba de Chi-cuadrada de razón de verosimilitudes (-2LL) del modelo final resultó estadísticamente diferente al inicial $\left(\chi^{2}=44.62, p<.000\right)$. Sobre la calidad del modelo, el parámetro pseudo R-cuadrado de Nagelkerke se situó en .44 puntos

Cuadro 4. Estimaciones de los parámetros tomando como referencia el grupo Ajustado-Estratégico

\begin{tabular}{lccccccc}
\hline & \multicolumn{3}{c}{$\chi^{2}$} & & & \multicolumn{2}{c}{ OR 95\% } \\
\cline { 7 - 8 } & $\mathrm{B}$ & Wald & $\mathrm{p}$ & $\mathrm{OR}$ & $\mathrm{Inf}$. & Sup. \\
\hline $\begin{array}{l}\text { Clínico-Evitativo } \\
\text { - Intersección }\end{array}$ & -0.812 & 3.71 & .054 & & & \\
$\begin{array}{l}\text { - Locus de control } \\
\text { parental }\end{array}$ & 1.257 & 9.28 & .002 & 3.51 & 1.56 & 7.89 \\
$\begin{array}{l}\text { - Satisfacción } \\
\text { - Cohesión }\end{array}$ & -1.029 & 6.94 & .008 & 0.36 & 0.17 & 0.77 \\
$\begin{array}{l}\text { Extremo-Pasivo } \\
\text { - Intersección }\end{array}$ & -1.474 & 12.71 & .000 & 0.23 & 0.10 & 0.51 \\
- Locus de control & 0.519 & 3.38 & .066 & & & \\
$\begin{array}{l}\text { parental } \\
\text { - Satisfacción }\end{array}$ & 0.423 & 2.31 & .128 & 1.53 & 0.88 & 2.63 \\
- Cohesión & -0.238 & 0.87 & .351 & 0.79 & 0.48 & 1.30 \\
\hline
\end{tabular}


por lo que se concluye que el modelo se encuentra ajustado y es válido al explicar el $44 \%$ de la varianza de los datos.

\section{DISCUSIÓN}

Este trabajo tuvo como objetivo analizar el estrés parental experimentado por las progenitoras de familias en riesgo y las estrategias de afrontamiento que despliegan para hacerle frente, así como explorar las relaciones entre el grado y el tipo de estrés y algunas dimensiones individuales y familiares relativas a la maternidad y la paternidad. En relación al primer objetivo, los análisis efectuados mostraron tres perfiles diferenciados: madres con un nivel moderado de estrés y estrategias de afrontamiento activas en el primer grupo, otro grupo con niveles clínicos de estrés y estrategias evitativas $\mathrm{y}$, por último, progenitoras con niveles extremos de estrés y pasivas. La aparición de tres grupos apoya la idea de que las familias en riesgo forman un colectivo heterogéneo con necesidades muy variadas, y que la intervención debe tener un carácter especializado..$^{24,30,31,39}$ De esta manera, el análisis tipológico del estrés parental y los procesos asociados al mismo ofrece claves que profundizan en el conocimiento de este tipo de familias, dejando patente la conveniencia de estudiar estas dimensiones desde este enfoque complementario. El grupo etiquetado como Ajustado-estratégico refuerza la tesis de Abidin ${ }^{2}$ en cuanto a que unos niveles moderados de estrés fomentan el uso de recursos que los progenitores poseen para afrontar satisfactoriamente las tareas asociadas a la paternidad, siendo la adecuada utilización de estas estrategias un factor clave para que las madres puedan hacer frente a las diferentes situaciones estresantes relacionadas con el cuidado de los menores. ${ }^{7}$ En la misma línea, tanto el grupo Clínico-evitativo como el Extremo-pasivo presentan estrategias de afrontamiento disfuncionales a mediano y largo plazo, si se tiene en cuenta que experimentar niveles altos de estrés y no hacerle frente de manera adecuada puede ser síntoma de alteraciones psicológicas y fomentar conductas maltratantes. ${ }^{8,32,33}$ Resulta fundamental en el ámbito de los S. S. C. identificar usuarias que presenten estas características para desarrollar intervenciones tanto clínicas como psicosociales precoces y ajustadas. ${ }^{31}$

El segundo objetivo de este estudio se centraba en las relaciones entre esta tipología y la percepción de las madres sobre sí mismas y sus familias. Comenzando con la eficacia parental cabe destacar la relación negativa que esta dimensión mantiene con el estrés parental en los análisis bivariados presentados en este trabajo, ${ }^{13,15}$ así como la baja percepción de eficacia que presentan las madres del grupo Clínico-evitativo. Con respecto a la satisfacción como progenitora, los resultados obtenidos apuntan a que los sentimientos emocionales positivos relacionados con el rol parental pueden reducir la sensación de estrés, ${ }^{16}$ de manera que si las mujeres del grupo Clínico-evitativo se sintieran más satisfechas como madres quedarían incluidas en el grupo Ajustado-estratégico. Siguiendo con las percepciones individuales que las madres tienen sobre sí mismas, el locus de control parental igualmente ha resultado relevante para la configuración de los grupos. Así, la percepción de control en cuanto a las tareas parentales fomenta unos adecuados niveles de estrés en situaciones adversas, facilitando además el uso de estrategias activas para hacerle frente en contraposición a la evitación de las mismas. ${ }^{18-20}$

Igualmente, el análisis de los lazos afectivos familiares ha aportado información imprescindible acerca de la tipología hallada. La cohesión familiar es la única dimensión que ha influido tanto en la explicación del perfil Clínico-evitativo como del Extremo-pasivo con respecto al más ajustado, de manera que, tal como apuntaban Vandsburger et al., ${ }^{28}$ el apoyo afectivo entre los miembros de la familia fomenta una satisfactoria adaptación de las madres frente al estrés $\mathrm{y}$, en circunstancias especialmente adversas, constituye una dimensión básica para amortiguar sus efectos perjudiciales. ${ }^{22,29}$ Además, y de acuerdo con Hardy et al., ${ }^{23}$ la cohesión familiar ha sido especialmente relevante a la hora de explicar la variedad de estrategias activas de afrontamiento que utilizan los diferentes miembros de la familia, ofreciendo así claves para comprender la diferencia entre el grupo estratégico y el pasivo. Por tanto, las dimensiones analizadas han demostrado ser de interés a la hora de intervenir con las familias en riesgo psicosocial.

Aunque este trabajo intenta claves útiles para las intervenciones que se realizan con las familias en riesgo, debemos apuntar una serie de limitaciones, la mayor parte de ellas relacionadas con la dificultad de contacto y al trabajo de campo necesario para realizar un estudio sobre este tipo de familias. Debido a ello el tamaño de la muestra de esta investigación es reducido (aunque debemos destacar que es superior al de muchas investigaciones de ámbito internacional en esta temática), y los resultados proceden de un único informante. Aun así, este estudio ofrece algunas implicaciones prácticas de cara a la intervención con estas madres, puesto que presenta una tipología por medio de dos instrumentos estandarizados y adaptados a nuestro país. ${ }^{41,43}$ Igualmente, las dimensiones psicológicas analizadas en este trabajo se han mostrado relevantes para el trabajo con las familias, siendo además susceptibles a la intervención. ${ }^{34-38}$ Por tanto, los resultados obtenidos profundizan en los distintos perfiles de las familias en riesgo así como en el conocimiento de sus necesidades, ofreciendo claves para intervenciones tanto clínicas como psicosociales con el objetivo de fortalecer los factores de protección que les pueden llevar a ser más resistentes y ajustadas ante las situaciones de adversidad que las rodean.

\section{REFERENCIAS}

1. Webster-Stratton C. Stress: A potential disruptor of parent perceptions and family interactions. J Clin Child Psychol 1990;19:302-312.

2. Abidin RR. The determinants of parenting behavior. J Clin Child Psychol 1992;21:407-412. 
3. Lazarus R, Folkman S. Stress, appraisal and coping. New York: Springer; 1984.

4. Abidin RR. Parenting Stress Index (Short Form). Odessa, USA: Psychological Assessment Resource; 1995.

5. Campos M, Iraurgui D, Velasco C. Afrontamiento y regulación emocional de hechos estresantes. Un meta-análisis de 13 estudios. Boletín Psicología 2004;82:25-44.

6. Boss PG. Family stress management. Newbury Park, USA: Sage; 1988.

7. McKelvey LM, Fitzgerald HE, Schiffman RF et al. Family stress and parent-infant interaction: The mediating role of coping. Infant Ment Health J 2002;23:164-181.

8. Riquelme A, Buendia J, Rodríguez MC. Estrategias de afrontamiento y apoyo social en personas con estrés económico. Psicothema 1993;5:83-89.

9. Crnic K, Low C. Everyday stresses and parenting. En: Bornstein MH (ed). Handbook of parenting: Vol. 5. Practical issues in parenting. Mahwah, USA: Lawrence Erlbaum; 2002.

10. Deater-Deckard K. Parenting stress and child adjustment: Some old hypotheses and new questions. Clin Psychol-Sci Pract 1998;5:314-327.

11. Costa NM, Weems CF, Pellerin $K$ et al. Parenting stress and childhood psychopathology: An examination of specificity to internalizing and externalizing symptoms. J Psychopathol Behav Assess 2006;28:113-122.

12. Feldman R, Eidelman AI, Rotenberg N. Parenting stress, infant emotion regulation, maternal sensitivity, and the cognitive development of triplets: A model for parent and child influences in a unique ecology. Child Dev 2004;75:1774-1791.

13. Jones TL, Prinz RJ. Potential roles of parental self-efficacy in parent and child adjustment: A review. Clin Psychol Rev 2005;3:341-363.

14. Jackson AP, Huang CC. Parenting stress and behavior among single mothers of preschoolers: The mediating role of self-efficacy. J Soc Serv Res 2000;26:29-42.

15. Sanders MR, Woolley ML. The relationship between maternal selfefficacy and parenting practices: implications for parent training. Child Care Health Dev 2005;31:65-73.

16. Carpenter A, Donohue B. Parental satisfaction in child abuse and neglect: A review of standardized measures. Aggress Violent Behav 2006;11:577-586.

17. Campis LK, Lyman RD, Prentice-Dunn S. The parental locus of control scale: Development and validation. J Clin Child Adolesc Psychol 1986;15:260-267.

18. Lyon B. Stress, coping, and health. En: Hill-Rice V (ed.). Handbook of stress, coping, and health. Washington DC: Sage Publications, Inc; 2000.

19. Lloyd T, Hastings RP. Parental locus of control and psychological well-being in mothers of children with intellectual disability. J Intellect Dev Dis 2009;34:104-115.

20. Treacy L, Tripp G, Baird A. Parent stress management training for attention-deficit/hyperactivity disorder. Behav Therapy 2005;36:223-233.

21. Amerikaner M, Monks G, Wolfe $P$ et al. Family interaction and individual psychological health. J Couns Dev 1994;72:614-620.

22. Ramírez GJ, Manongdo JA, Cruz-Santiago M. The family as mediator of the impact of parent-youth acculturation/enculturation and innercity stressors on Mexican American youth substance use. Cultural Diversity Ethnic Minority Psychology 2010;16:404-412.

23. Hardy DF, Power TG, Jaedicke S. Examining the relation of parenting to children's coping with everyday stress. Child Dev 1993;64:1829-1841.

24. Krohne HW. Individual differences in coping. En: Zeidner M, Endler NS (eds). Handbook of coping: Theory, research, applications. Nueva York: Wiley; 1996.

25. Lorence B, Hidalgo MV, Dekovic M. Adolescent adjustment in at-risk families: The role of psychosocial stress and parental socialization. Salud Mental 2013;36:49-57.

26. Rodrigo MJ, Máiquez ML, Martín JC et al. Preservación familiar. Un enfoque positivo para la intervención con familias. Madrid: Pirámide; 2008.

27. Anderson LS. Predictors of parenting stress in a diverse sample of parents of early adolescents in high-risk communities. Nurs Res 2008;57:340-350.
28. Vandsburger E, Harrigan M, Biggerstaff M. In spite of all, we make it: Themes of stress and resiliency as told by women in families living in poverty. J Fam Soc Work 2008;11:17-35.

29. Zolkoski SM, Bullock LM. Resilience in children and youth: A review. Child Youth Serv Rev 2012;34:2295-2303.

30. Ayoub CC, Willett JB, Robinson DS. Families at risk of child maltreatment: Entry-level characteristics and growth in family functioning during treatment. Child Abuse Negl 1992;16:495-511.

31. Rodríguez G, Camacho J, Rodrigo MJ et al. Evaluación del riesgo psicosocial en familias usuarias de servicios sociales municipales. Psicothema 2006;18:200-206.

32. Stith SM, Liu T, Davies LC et al. Risk factors in child maltreatment: A meta-analytic review of the literature. Aggress Violent Behav 2009;14:13-29.

33. Hillson MC, Kuiper NA. A stress and coping model of child maltreatment. Clin Psychol Rev 1994;14:287-299.

34. Begle AM, Dumas JE. Child and parental outcomes following involvement in a preventive intervention: Efficacy of the PACE program. J Prim Prev 2011;32:67-81.

35. Bloomfield L, Kendall S. Parenting self-efficacy, parenting stress and child behaviour before and after a parenting programme. Prim Health Care Res Dev 2012;1:364-372.

36. Smith $M$. Measures for assessing parenting in research and practice. Child Adol Ment H-UK 2011;16:158-166.

37. Padilla S, Máiquez ML, Rodrigo MJ. Influencia de las características familiares sobre el estrés parental en familias en riesgo psicosocial. Oviedo, España: XI Congreso Internacional de Infancia Maltratada; 2012.

38. Padilla S, Máiquez ML, Rodrigo MJ. Evaluación de un programa domiciliario: Crecer felices en familia. Oviedo, España: XI Congreso Internacional de Infancia Maltratada; 2012.

39. Hidalgo MV, Menéndez S, Sánchez J et al. Intervention with at-risk families: Contributions from a psycho-educational perspective. Psychol Spain 2010;14:48-56.

40. Observatorio de la Infancia en Andalucía. Pobreza y desigualdad. Cifras y Datos $n^{\circ}$ 10. Sevilla, España: Consejería de Salud y Bienestar Social; 2013.

41. Díaz-Herrero A, Brito A, López JA et al. Estructura factorial y consistencia interna de la versión española del Parenting Stress Index -short form. Psicothema 2010;22:1033-1038.

42. Carver CS. You want to measure coping but your protocol's too long: Consider the Brief COPE. Int J Behav Med 1997;4:92-100.

43. Crespo M, Cruzado JA. La evaluación del afrontamiento: adaptación española del cuestionario COPE con una muestra de estudiantes universitarios. Anál Modif Conducta 1997;23:797-830.

44. Johnston C, Mash EJ. A measure of parenting satisfaction and efficacy. J Clin Child Psychol 1989;18:167-175.

45. Olson DH, Portner J, Lavee, Y. FACES III. St Paul, Minnesota, USA: University of Minnesota; 1985.

46. Pardo A, Ruíz MA. SPSS. Guía para el análisis de datos. Madrid: McGraw-Hill; 2005.

47. Pérez C. Técnicas de análisis multivariante de datos. Aplicaciones con SPSS. Madrid: Pearson-Prentice Hall; 2004.

48. Henry DB, Tolan PH, Gorman-Smith D. Cluster analysis in family psychology research. J Fam Psychol 2005;19:121-132.

49. Tabachnick BG, Fidell LS. Using multivariate statistics. Quinta edición. Boston: Pearson Education; 2007.

50. Jovell AJ. Análisis de regresión logística. Madrid: Centro de Investigaciones Sociológicas; 1995.

51. Ortega M, Cayuela A. Regresión logística no condicionada y tamaño de muestra: Una revisión bibliográfica. Rev Esp Salud Pública 2002;76:85-93.

Artículo sin conflicto de intereses 